\title{
Regional Therapies for Advanced Cancer: Update for 2016
}

\author{
Kiran K. Turaga, MD, MPH ${ }^{1}$, T. Clark Gamblin, MD, MS, MBA ${ }^{2}$, H. Richard Alexander, MD, PhD ${ }^{3}$, \\ Robert Edwards, $\mathrm{MD}^{4}$, and David L. Bartlett, $\mathrm{MD}^{5}$
}

${ }^{1}$ Division of Surgical Oncology, Department of Surgery, Medical College of Wisconsin, Milwaukee, WI; ${ }^{2}$ Department of Surgery, Medical College of Wisconsin, Milwaukee, WI; ${ }^{3}$ Department of Surgery, University of Maryland Medical Center, Baltimore, MD; ${ }^{4}$ Department of Obstetrics and Gynecology, University of Pittsburgh, Pittsburgh, PA; ${ }^{5}$ Department of Surgery, University of Pittsburgh, Pittsburgh, PA

A decade since its inception, the Tenth International Symposium on Regional Therapies, held at Clearwater Beach, Florida, in 2015, set the stage for a quiet revolution. The universality of human suffering and disease and the untiring efforts of researchers, physicians, patients, and healthcare providers brought together many such individuals in a common mission to highlight the role of regional therapies in patients with advanced malignancies. This supplement attempts to provide readers with a flavor of the impressive research presented, but it cannot capture the camaraderie, debates, and scientific banter that were shared.

In this issue, selected articles are categorized on broad themes. Selection of patients undergoing cytoreductive surgery and hyperthermic intraperitoneal chemotherapy (HIPEC) is critical to ensure successful outcomes. Sammartine et al. describe a common platform to be able to do so. ${ }^{1}$ They describe a Web-based tool that can accurately capture the peritoneal carcinomatosis index (PCI) score and the completeness of cytoreduction (CC) score. This attempt to accurately and objectively measure disease may be the first step in selecting patients appropriately. Another tool in selecting patients appropriately is diagnostic laparoscopy, which is the feature of the article by Marmor et al. ${ }^{2}$ Among 145 patients, $81.6 \%$ of whom had previous abdominal operations, the authors describe the ability to perform successful laparoscopy with four intraoperative complications (two enterotomies). Although it is a safe

\footnotetext{
(C) Society of Surgical Oncology 2016
}

First Received: 17 December 2015;

Published Online: 5 January 2016

K. K. Turaga, MD, MPH

e-mail: kturaga@mcw.edu tool, one has to be aware of the invasive nature of such staging.

Regardless of the advances in detecting and documenting the burden of disease, the tumor's histologic characteristics often determine patient outcome. In articles from Kusamura and Ihemelandu, proliferative markers such as Ki-67 as well as signet ring cells and adenocarcinoid features are utilized to stratify patients on the basis of their outcomes. ${ }^{3,4}$ Using conditional tree inference, they were able to identify patients with epithelioid malignant mesothelioma who could be stratified into good prognostic characteristics $\quad(\mathrm{Ki}-67<9 \%, \quad \mathrm{PCI} \leq 17) . \quad$ Similarly, extensive cytoreduction showed a marginal survival benefits for patients with adenocarcinoid and signet ring cell morphology with mucinous adenocarcinomas with peritoneal disease (5-year survival, 15 and $22 \%$, respectively).

Doud and colleagues examine an important issue of diverting ostomies for patients undergoing cytoreductive surgery and HIPEC. ${ }^{5}$ In such patients, only a humbling $26 \%$ underwent reversal after a temporary diverting ostomy was placed. The 30-day mortality of the reversal was $4.7 \%$. Sugarbaker et al. describe a technique of avoiding a temporary loop ileostomy during a pelvic peritonectomy by using longer length of rectum and describe a reduction in diverting ostomy rate, to 7 from $50 \%{ }^{6}$

Two articles explore the morbidity of cytoreductive surgery and intraperitoneal chemotherapy, both of which have implications for older patients. Patients aged $\geq 65$ years were $60 \%$ more likely to experience morbidity (30-day rate $44 \%$, mortality $3.2 \%))^{7,8}$ The presence of increasing comorbid factors significantly increased the overall morbidity of the procedure in this population. Contrary to the perception that the procedure is highly morbid, requiring intensive care unit (ICU) stay, $23 \%$ of patients did not require ICU stay in the 
study by Mogal et al. ${ }^{7}$ Those that did require ICU stay were more likely older, had a poorer performance status, or underwent larger resections.

As immunotherapy and immune checkpoint inhibitors offer an interesting look at managing advanced malignancies, Thomas et al. examined the role of the tumor microenvironment in malignant mesothelioma. ${ }^{9}$ After examining the serum and ascites of patients with malignant mesothelioma, IL- 8 and MIP- $\beta$ were found to be present at higher levels, with a profile distinct from either inflammation or trauma. Although not currently applicable to the therapeutic environment, it raises exciting future possibilities.

Our ability to utilize patients as effective stakeholders relies on converting scientific data to patient-centered data. Mogal et al. examine the concept of conditional survival for appendiceal carcinomas. ${ }^{10}$ Although overall survival is an informative metric, it is often irrelevant to patients who have already lived a certain period of time. The utility of conditional survival lies therein-for instance, the conditional survival for those with highgrade disease at 10 years is $42 \%$ as opposed to a 10-year survival of $26 \%$.

The last article reports on a case series of patients with uterine sarcomatosis from laparoscopic morcellation techniques during hysterectomies. ${ }^{11}$ In our efforts to advance science and surgery, it is extremely important to tread with caution, as this case series demonstrates. The adoption of novel technology is not always closely regulated, which allows it to be nimble, yet quality review is essential to continue our trend to forward progress.

This year, like many of the previous years, demonstrates our constant desire to get better. Better science, better surgery, and better health are universal goals for surgical oncologists across the world. We invite you to join us as we develop and disseminate a platform for sharing and improving the management of advanced malignancies.
DISCLOSURE The authors declare no conflict of interest.

\section{REFERENCES}

1. Sammartine P, et al. Computerized system for staging peritoneal surface malignancies. Ann Surg Oncol. 2015. doi:10.1245/ s10434-015-4966-5.

2. Marmor R, et al. Laparoscopy is safe and accurate to evaluate peritoneal surface metastasis prior to cytoreductive surgery. Ann Surg Oncol. 2015. doi:10.1245/s10434-015-4958-5.

3. Kusamura S, et al. The role of Ki-67 and pre-cytoreduction parameters in selecting diffuse malignant peritoneal mesothelioma (DMPM) patients for cytoreductive surgery (CRS) and hyperthermic intraperitoneal chemotherapy (HIPEC). Ann Surg Oncol. 2015. doi:10.1245/s10434-015-4962-9.

4. Ihemelandu $\mathrm{C}$, et al. Clinicopathologic and prognostic features in patients with peritoneal metastasis from mucinous adenocarcinoma, adenocarcinoma with signet ring cells and adenocarcinoid of the appendix treated with cytoreductive surgery and perioperative intraperitoneal chemotherapy. Ann Surg Oncol. 2015. doi:10.1245/s10434-015-4995-0.

5. Doud A, et al. Stoma creation and reversal after cytoreductive surgery with hyperthermic intraperitoneal chemotherapy. Ann Surg Oncol. 2015. doi:10.1245/s10434-015-4674-1.

6. Sugarbaker $\mathrm{P}$, et al. Avoiding diverting ileostomy in patients requiring complete pelvic peritonectomy. Ann Surg Oncol. 2015. doi:10.1245/s10434-015-4961-x.

7. Mogal $\mathrm{H}$, et al. Routine admission to ICU after cytoreductive surgery (CRS) and heated intraperitoneal chemotherapy (HIPEC): not always a requirement. Ann Surg Oncol. 2015. doi:10.1245/ s10434-015-4963-8.

8. Peters $\mathrm{M}$, et al. Age related morbidity and mortality with cytoreductive surgery and intraperitoneal chemotherapy. Ann Surg Oncol. 2015. doi:10.1245/s10434-015-4624-y.

9. Thomas $\mathrm{P}$, et al. Malignant peritoneal mesothelioma: characterization of the inflammatory response in the tumor microenvironment. Ann Surg Oncol. 2015. doi:10.1245/s10434-015-4965-6.

10. Mogal $\mathrm{H}$, et al. Conditional survival after cytoreductive surgery (CRS) with heated intraperitoneal chemotherapy (HIPEC) for low- and high-grade appendiceal primaries. Ann Surg Oncol. 2015. doi:10.1245/s10434-015-4821-8.

11. Sugarbaker P, et al. Cytoreductive surgery and HIPEC as a treatment option for laparoscopic resection of uterine leiomyosarcoma with morcellation: early results. Ann Surg Oncol. 2015. doi:10. 1245/s10434-015-4960-y. 